Témoigner Témoigner. Entre histoire et mémoire

Getuigen Revue pluridisciplinaire de la Fondation Auschwitz

$123 \mid 2016$

Traduire le témoignage

\title{
Paul Sobol et la résilience : revivre après Auschwitz
} Propos recueillis par Jean-Louis Rouhart (extraits)

\section{Paul Sobol et Jean-Louis Rouhart}

\section{(2) OpenEdition}

Journals

Édition électronique

URL : https://journals.openedition.org/temoigner/5440

DOI : $10.4000 /$ temoigner. 5440

ISSN : 2506-6390

Éditeur :

Éditions du Centre d'études et de documentation Mémoire d'Auschwitz, Éditions Kimé

Édition imprimée

Date de publication : 1 octobre 2016

Pagination : 168-181

ISBN : 987 2-9600926-4-6

ISSN : 2031-4183

Référence électronique

Paul Sobol et Jean-Louis Rouhart, «Paul Sobol et la résilience : revivre après Auschwitz », Témoigner. Entre histoire et mémoire [En ligne], 123 | 2016, mis en ligne le 02 novembre 2021, consulté le 04 novembre 2021. URL : http://journals.openedition.org/temoigner/5440 ; DOI : https://doi.org/10.4000/ temoigner.5440 


\section{Paul Sobol et la résilience Revivre après Auschwitz}

Jean-Louis Rouhart : Paul Sobol, avant d'aborder le thème de la résilience, je vous propose de retracer d'abord le parcours que vous avez effectué depuis l'enfance jusqu'à votre libération des camps et votre retour à Bruxelles en 1945.

Paul Sobol : J'ai décrit cet itinéraire dans mon autobiographie intitulée « Je me souviens d'Auschwitz Del'étoile de shérif a la croixde vie „Ce livre est paru en 2010 aux Éditions Racine. Si vous le désirez, je peux vous en lire un extrait.

\section{Je vous en prie.}

«Je suis néà Paris. Mes parents sont juifs sans être pratiquants. Nous nous installons à Bruxelles en 1927. Lorsque la guerre éclate, nous continuons à vivre peu ou prou comme avant. En 1940,j’arrête l'école à 14 ans pour aider financièrement ma famille. Mon père nous rement ainscils gique. Nous portons rétolle jaune sur nos vêtements. En 1942, après une terrible rafle dans le quartier du Midi, mon père décide de nous faire entrer en clandestinité. J'ai une nouvelle carte d'identité, un nouveau nom. Je fais du sport et me lie d'amitiéavec un groupe de jeunes catholiques parmi lesquels se trouve une bellejeune fille Nelly Je tombe ane belleje pille Nelly. Jetombe juin 1944 . Deux jours avant juin 1944, 37 jours avant la libéra-

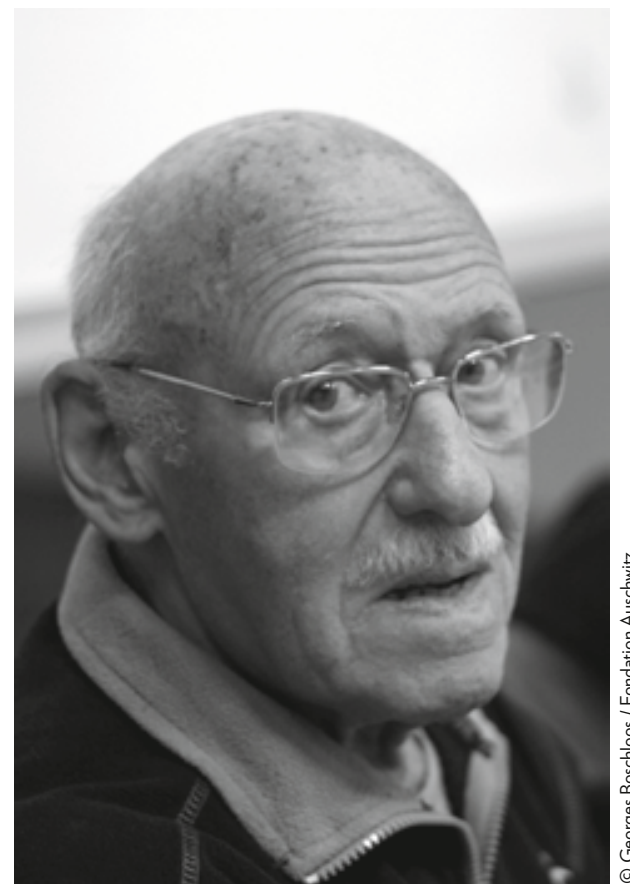

tion de Bruxelles, nous sommes arrêtés en pleine nuit par la Gestapo (après avoir été dénoncés) et envoyés à la caserne Dossin à Malines. Là-bas, je reçois un colis de vivres de Nelly : elle s'était renseignée et avait appris mon arrestation. Dans le colis, je trouve une petite photo d'elle que je garde précieusement. Nous sommes envoyés à Auschwitz : mon père, ma mère, ma sœur Betsy, mon frère David et moi-même. À notre arrivée, les hommes et les femmes sont séparés. Mon père, David et moi restons ensemble. Peu de jours après, David est emmené Nous ne le reverrons jamais. Nous sommes humiliés physiquement, affamés, tondus et tatoús comme des animaux semaux Je me fais passer pour un menuisier et je decore des petites boites. Mon Kapo qui les vend plus cher me protège et me donne un peu de nourriture. Je retrouve mon père un dimanche sur deux sur la place d'appel. Ce que nous vivons chaque jour dépasse l'entendement: les coups, la souffrance de ceux qui ne résistent pas, le froid, la peur, la faim, le spectacle quotidien de la mort. Nous apprenons la libération de Bruxelles avec joie. Je tiens le coup grâce à la petite photo de Nelly pliée en huit, l'espoir de la revoir.»

Cette photographie vous a donc accompagné durant toute votre détention. Elle vous a donné la force de ne pas sombrer dans le néant. Elle fait partie sans nul doute des ressources externes que vous avez pu exploiter pour survivre et que vous mentionnez dans votre ouvrage (Sobol, 2010, p. 152)

Oui, c'est grâce à elle que j’ai surmonté l'adversité et aussi parce que j’ai utilisé les valeurs que j’avais en moi, notamment un certain talent pour dessiner.

Sije comprends bien, le fait d'avoir poursuivi un but bien précis - survivre pour pouvoir un jour revoir l'être aimé - a donné un sens à votre vie durant ces longs mois et vous a permis de survivre dans un milieu mortifère ; la proximité du père à certains moments vous a apporté une autre certitude et a constitué un autre point de repère. Quant au dessin, non seulement il vous a permis de sortir du lot et vous a sans doute sauvé la vie, mais en tant qu'expression de fantaisie dans le monde uniforme et monotone des camps, il vous a peut-être aussi permis d'oublier pour quelques instants la dure rélité du cal in du camp dAuschwits sion fait in famille unie), quivous ont permis dacquerir duran votre enfance les ressources internes indispensables pour reagir face aux agressions de lexistence, et si on ajoute une bonne " organisation " pour résoudre le problème quotidien de la faim, nous aurons, je crois, défini les éléments qui ont constitué votre stratégie de survie au camp d'Auschwitz. Comme vous le savez, cette capacité à résister au traumatisme de la captivité est désormais connue en psychologie sous le nom de « résilience ». C'est le premier aspect de la résilience, le second étant la capacité à se reconstruire après le trauma. Nous reviendrons plus tard sur cet autre aspect important, car nous ne sommes pas encore au bout de votre parcours dans les camps.

En effet. (Il poursuit sa lecture). «En janvier 1945, lévacuation du camp d’Auschwitz commence. Je perds de vue mon père. Je ne le reverrai plus. Nous marchons 
dans un froid glacial avec la menace permanente d'être tués par les soldats. Nous faisons 200 kilomètres en trois jours entassés comme des bêtes dans un train de marchandises. La folie guette les hommes dans le wagon. On meurt de faim, de soif. Nous arrivons enfin au bout de 6 à 7 jours à Dachau. Ceux qui ont survécu sont mis en quarantaine. Pas de travail à faire, pas de nourriture. Nous apprenons la libération du camp d'Auschwitz. Quatre jours après, on nous donne à boire, à manger et nous repartons dans un camion pour un sous-camp de Dachau où nous travaillon comme des forcenés, ce que nous sommes. Ià je rencontre une femme rencontré con à la caserne Dossin. Elle na pas de nouvelles de ma mère et de ma sœur. Elle me donne un peu de vivres en cachette. Nous repartons dans un train. Nous profiton d’un bombardement pour nous échapper. Je m’enfuis avec un Français. Un paysan allemand nous met en joue avec son fusil, mais ne tire pas quand nous prenons nos jambes à nos cous. Dans un village, nous rencontrons des prisonniers de guerre français. Ils travaillent dans des fermes durant la journée et le soir, un soldat allemand les enferme dans le théâtre du village qui fait office de prison où ils disposent d'un confort incroyable et de victuailles à volonté. Nous prenons la place de deux prisonniers qui restent dormir dans les fermes. Notre premier vrai repas manque de nous tuer : trop gras, trop riche pour nos pauvres corps. Et quelques jours après, le ler mai 1945, les Américains sontlì Nous sommes rapatriés vers la France dans les mêmes was es mêmes wagons qui nous y avaient anenés mais dans dautesconditions! Je sui pris en charge par la Croix-Rouge française et belge qui me rapatrie à Bruxelles.»

Manifestement, durant l'espace de temps compris entre votre évacuation du camp d'Auschwitz et votre libération, vous avez à nouveau frôlé la mort, et ce, à plusieurs reprises. Comment expliquez-vous votre survie?

Depuis des années, je me pose la question : «Pourquoi moi ? Pourquoi suis-je revenu et non mes parents ni David, mon jeune frère ?» Une chose est sûre, si j’a survécu, c'est grâce à un ensemble de petits faits miraculeux qui, à des moment fatidiques mont permis de sortirvivant del'enfer des camps, comme le fait de pents à Nelly, sa photo que jai pu garder tout au long de ma captivité, ma décision rapide de me faire passer pour un menuisier ou encore la rencontre avec les prisonniers de guerre français alors que j’étais affamé... Il y a eu le facteur chance, l'instinct de survie, la volonté égoïste de survivre [...]

II semble que, face à différentes situations de risque, vous ayez pu, comme d'autres rescapés, développer une forme de résilience qui vous a permis de résister, de vous adapter, de vous fortifier et ainsi de réagir au traumatisme quotidien (Colchado). Il est possible également que vous possédiez le "gène du surhomme " qui facilite le transport de la sérotonine, un neuromédiateur qui lutte conte les émotions dépressives et joue un rôle détermin déterné aura eteutlepar lisution, intérieure qui vous a permis de mener de façon remarquable votre vie après le camp. Votre expérience du camp de la mort aura été un chemin initiatique qui vous aura donné une force de vivre extraordinaire pour le restant de votre vie (Raulier, 2010, 216 sq.)

Oui, l'expérience des camps a permis de révéler ce que j’avais potentiellement de positif en moi pour survivre et revenir. Mon expérience comme « esclave des nazis » m’a permis de rester dans le monde des vivants, celui où on est simplement «esclave de la liberté».

\section{Qu'entendez-vous exactement par «être esclave de la liberté »?}

C'est pouvoir travailler librement, organiser soi-même sa vie en étant auteur de son destin, maître de sa destinée. Auschwitz m’aura appris aussi à vivre chaque jour de ma vie comme si c'était le dernier. Comme je lécris dans mon livre, «l'important dans la vie, c'est de savoir où l'on veut aller. C'est la motivation essentielle pour agir et pour être en accord avec soi-même. Cela tend les ressorts de la volonté et maintient l'enthousiasme dans l'effort. Cela, je l'ai appris grâce à Auschwitz.» (Sobol, 2010, p. 153) Il est vraisemblable que la créativité et l'imagination débordante, dont j’a fait preuve par la suite, ont été fustigées par l'expérience des camps.

\section{Parlons à présent de la période qui a suivi votre retour à Bruxelles.}

(Paul Sobol reprendla lecture de son texte) : «À Bruxelles, c'est la fin du cauchemar. J'appelle un de mes anciens amis qui m'héberge car je n'ai plus de famille. Je tombe malade, une vilaine pleurésie. Je revois enfin Nelly. Je retrouve ma sœur Betsy. Elle n’a pas de nouvelles de ma mère. Je pars en maison de repos grâce à l'intervention d'un membre du parti socialiste de l'époque, puis dans une villa en Suisse, envoyé par l'armée belge. Je rentre à Bruxelles avec un peu d'argent reçu de l'armée. Je loue une chambre et cherche un travail. Je rencontre alors Jacques Meert le secrétaire particulier ducardinal Joseph Cardijn, connupour avoir mis surpied les « Jeunesses ouvrières chrétiennes ». Il me trouve un travail, me pousse à me faire baptiser et convainc les parents de Nelly que je suis un bon garçon, et un bon garçon pour leur

On pourrait croire que votre histoire se termine comme un conte de fées, par un happy end. Pourtant, dans votre autobiographie « Je me souviens d'A uschwitz... De l'étoile de shérif à la croix de vie ", vous évoquez aux chapitres "Liberté et reconstruction » et «Survivre après Auschwitz » les difficultés que vous avez rencontrées à votre retour.

C'est exact. En plus de la pleurésie, j’ai eu la gale et une plaie inquiétante sur ma jambe droite qu'il a fallu soigner. J'ai dû progressivement me faire à l'idée que je ne questionnait sur ce que j'ai vécu dans les camps nazis. Les Belges avaient souffert 


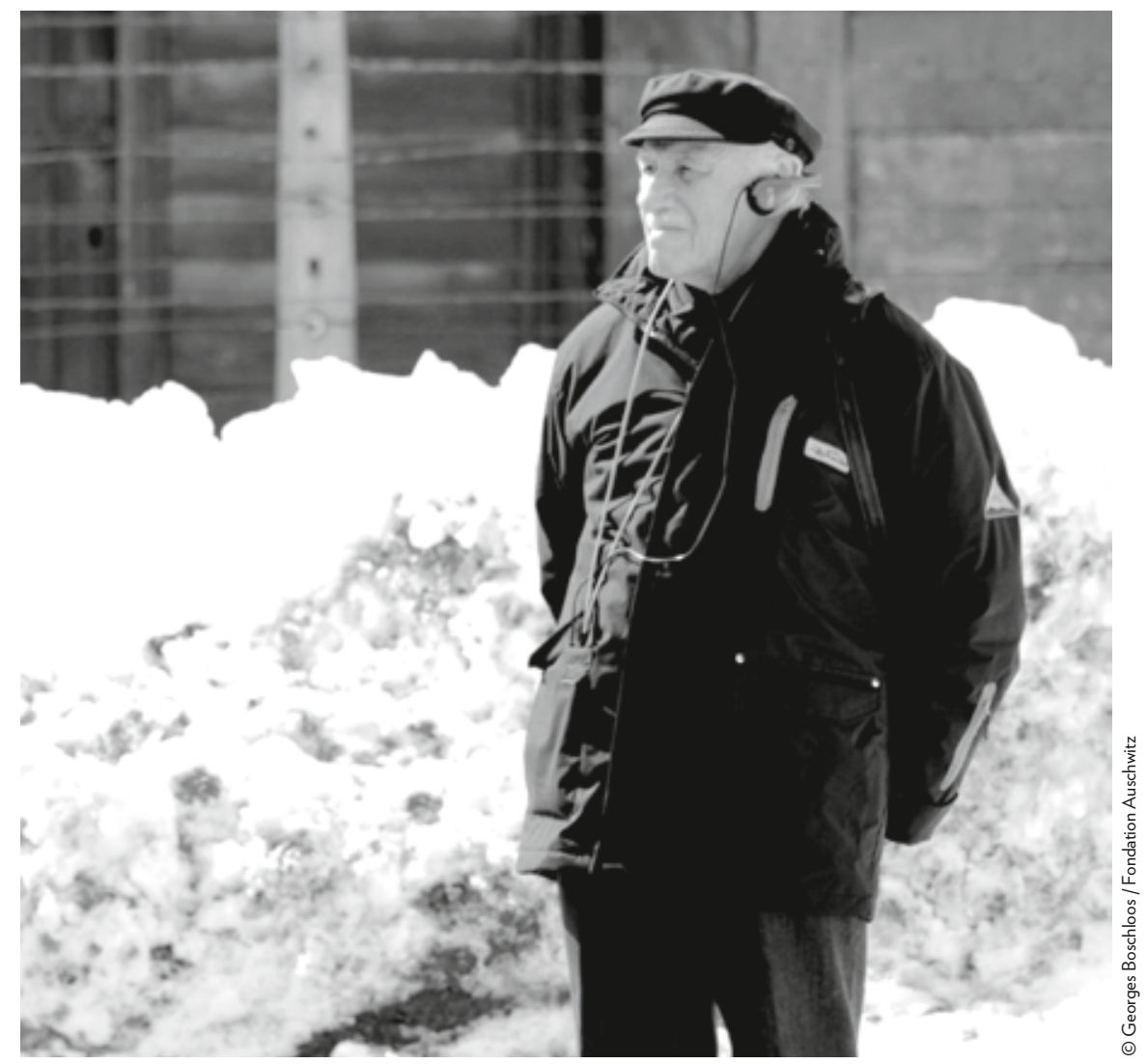

de faim, de froid, de l'Occupation. Ils ne pensaient qu’à l'avenir et voulaient oublier la guerre. J'avais honte de leur parler de ce que javais été: un esclave pour les nazis. la guerre. J avais honte de leur parler de ce que javais eté : un esclave pour les nazis. Je ne pouvais leur dire comment ils m'avaient détruit, commej'avais été contraint renier toutes valeurs humaines pour survivre. Je ne leur montrais même pas mon tatouage B-3635. Ils n'auraient pas compris. Ou bien, ils n'auraient pas voulu savoir Comme il n'y avait aucune écoute, je me suis tu. J'étais tout seul, sans famille et sans logement. Personne ne s'occupait de moi. Mes demandes pour obtenir la naturalisation belge n’aboutissaient pas. Comme je n’avais pas fait d'études à cause de la guerre, je n’avais ni diplômes, ni formation, ni plan d’avenir.

Cette situation a été vécue douloureusement par nombre d'autres déportés rescapés. Boris Cyrulnik, entre autres, en parle dans ses ouvrages consacrés à la résilience. Condannés au mutisme sálectif a fin de ne pas choquer et privés du soutien sécurisant Condamnés au mutisme sér de leur entourage, certains rescapés, selon lui, n'ont pas pu tenir les images de l'horreur
à distance. Celles-ci sont revenues comme des éclairs douloureux et se sont imposées à leur conscience muette. Cette souffrance les a empêchés de reprendre une place dans la société, en a fait des épouvantails isolés, réduits au silence et souffrant en secret (Cyrulnik, 2008, p. 221). Mais vous, Paul Sobol, malgré toutes ces difficultés, vous avez pu rebondir, vous reconstruire et retrouver progressivement une place dans la société.

Comme je vous le disais, il y a un moment, j’ai eu la chance d'avoir été pris en charge par des institutions, puis d’avoir rencontré Jacques Meert, le secrétaire par-

Apparemment, lors du processus de résilience qui s'est développé à votre retour des camps, cet homme a joué le rôle du tuteur signifiant, il a été le personnage « initiateur » (Bacqué). Il aura été la main tendue, le facteur de résilience qui vous aura permis de renouer le lien social et de reprendre le développement qui avait été infléchi par votre blessure (Cyrulnik, 2004, p. 17, p. 225)

N’oublions pas Nelly, grâce à qui j’ai pu fonder une famille. Cétait mon souhait e plus cher, avoir une nouvelle famille pour remplacer celle que j'avais perdue. Cela aura été ma victoire sur les nazis. Nous avons eu, Nelly et moi, deux enfants, un garçon et une fille [...].

Avez-vous souvent parlé de votre déportation dans les camps à vos enfants?

Jamais. J’ai toujours voulu les protéger. Ils sont catholiques comme leur mère et lorsqu'ils me posaient des questions telles que : «pourquoi n’ai-je pas deux grandmères, deux grands-pères, pas plus de cousins ou de cousines, d'oncles, de tantes de ton côté ? Pourquoi ce numéro tatoué sur ton bras gauche?» Chaque fois, jéludais les questions par des réponses sibyllines. En famille, on ne parlait jamais de la guerre. Ma vie était devant moi : travailler, voyager, plonger... Et non derrière et je ne voulais

Boris Cyrulnik parle aussi du dilemme devant lequel sont placés les résilients : témoigner pour eux, c'est transmettre un traumatisme, se taire, cest transmettre l'angoisse du gner pour eux, cest transmettre un traumatisme, se taire, cest transmetre langoisse du silence (Cyrulnik, 2010, p. 12). Selon lui, il est plus commode pour les résilients de dénier les événements traumatisants, car ce déni, s'il n'efface pas l'empreinte du traumatisme dans la mémoire biologique, empêche de ressasser. L'évitement de l'affrontement avec la réalité douloureuse entraîne une absence de conflictualité et constitue de surcroît un avantage relationnel, parce qu'on constate que le déni aide à façonner une personnalité aux relations agréables (Cyrulnik, 2004, p. 196 sq.) [...]. Mais revenons à votre parcours professionnel et sportif après votre mariage.

J'ai eu une vie professionnelle longue et fort captivante. Un hasard m'a mis sur la voie de la réclame, l'ancêtre de la publicité, du marketing et de la communication. J'ai fondé de nombreuses sociétés et ai engagé des personnes compétentes pour les 
gérer, moi, le créatif n’ayant que des idées! Mon parcours a étéjalonné de nombreux succès mais également d'échecs.

De manière assez significative, vous renoncez très rapidement à travailler comme employé. Vous êtes un des premiers à fonder votre propre agence de publicité en Belgique et par la suite, vous continuez votre parcours professionnel en tant qui indépendant.

Je n'aurais pas supporté d'être considéré comme un numéro, d'être l'esclave d'une pointeuse. Cela m’aurait trop rappelé Auschwitz et mon tatouage B3635. Ma liberté m’aurait manqué cruellement.

On peut supposer que cette volonté d'indépendance s'inscrit, elle aussi, dans la ligne du processus de résilience, dans la mesure où tout ce qui rappelait la déportation l'obéissance aveugle, la dépendance d'autres personnes, devait nécessairement être perçu comme des éléments négatifs, être rejeté et rangé dans les mauvais souvenirs rattachés à la vie d'avant la résurrection, la renaissance. Faut-il voir dans votre parcours plutôt les succès ou les échecs ?

Il faut envisager les deux.

Apparemment, c'est dans le secteur de la distribution du matériel et d'équipements de plongée ainsi que dans la vente d'articles se rapportant aux activités nautiques que vous avez enregistré le plus de succès.

Oui. J’ai effectivement vendu du matériel de sports nautiques et des équipements de plongée. J’ai édité également des revues centrées sur la plongée, fondé des centre de fitness et de plongée, réalisé des films documentaires sur la vie sous-marine. On m’a appelé le pionnier du tourisme sous-marin, parce que j’ai élaboré avec des tours opérateurs et des compagnies aériennes des projets de création de clubs de tours opérateús sur des compagnies átiennes des projets de création de clubs de vacances axés sur la plongée et mis sur pied des centres de plongée dans des hôtel en Turquie et en Egypte. La plongée a toujours été pour moi une grande passion.
En 2015, j’ai reçu mon diplôme de 55 ans de monitorat national!

\section{Peut-on parler aussi des échecs?}

Je me suis parfois entouré d’associés malhonnêtes ou peu scrupuleux contre qui j’ai dû me battre en justice. Dans certains cas, mes idées étaient bien reçues, mais la réalisation était compromise à cause de négligences de tiers.

\section{Et puis, il y a aussi un tournant, un moment critique dans votre carrière.}

En 1963, je prends de mauvaises décisions en fusionnant avec une agence française de publicité qui se révèle être constituée d'escrocs. Ils m’éjectent de ma propre société et je suis en procès pendant trois ans. Je gagne à chaque fois, mais ils sont très procéduriers et pour finir, je perds tout: ma société, ma voiture, mes clients et mon boulot! Ma femme Nelly ne comprend pas ce qui m’arrive. Je ne la tenais pas au courant de mes affaires, elle croyait que tout se passait pour le mieux pour nous. Je protégeais ma famille, voulant résoudre mes problèmes seul, comme un homme d'affaires... sans aucune expérience! Lorsque j'explique à Nelly dans quelle situation nous sommes, elle est sous le choc, traumatisée. Je vis très mal cette descente aux enfers. Il n'y aque des problèmes à tésoudrequi, de plus, ne sont tence. Mon avenir est entre les mains de mes avocats!

\section{Que se passe-t-il ensuite?}

Un ami me donne une voiture, une véritable épave destinée à la casse et un autre copain, Georges, le patron de la firme C., une société de publicité lumineuse, m’engage comme directeur commercial. C'est déjà mieux que rien ! La voiture n’a rien à voir avec ma Porsche et ce boulot de commercial n'est pas mon truc. C'est la première fois dans ma vie que je me sens au plus bas, au fond du trou, que je perds tout espoir d'en sortir. Et que je pense au suicide! Même dans les camps, jamais je n'avais pensé à mourir. Javais alors la petite photo de Nelly pour me remonter le rir. Javais alors la petite photo de Nelly pour me remonter le moral et ma volonté de survivre avec l'espoir de la revoir.

\section{À ce moment, on peut dire que le processus de résilience, entamé depuis des années,} est gravement compromis

Je pense en finir sur la route vers Waterloo pour rejoindre Robert R., qui lui aussi a des problèmes. Je me dis : avec cette vieille voiture, si je me jette contre un arbre, ça paraîtra normal et comme je suis bien assuré, Nelly et les enfants pourront continuer à vivre normalement tout en pensant à moi ! Cela se transforme en obsession dans mon esprit. Au volant, plus je roule, plus je roule vite en cherchant l'arbre idéal! Soudain, je me revois à Auschwitz. A mon arrivée, encore en quarantaine, pour pouvoir être un tout petit peu mieux traite, je me suis proposé comme menuisier alors que je ne connaissais pas ce métier. A ce moment-là, j’ai risqué ma vie. Finalement, j'ai trouvé divers petits boulots et je suis devenu le valet des nazis ! Et aujourd'hui sur cette route, je joue ma vie et celle de ma famille «juste» pour une question d'argent? [...] J'ai encore une longue vie devant moi. Je dois m'en sortir. Je ne peux pas laisser Nelly. Je me dois de lui prouver que je suis toujours aussi fort et combatif quavant, tant pour elle que pour notre famille ! Je reprends alors une conduite normale en me jurant que c'est la dernière fois que je roule avec cette vieille voiture. Le lendemain, à Bruxelles, je cours acheter une bonne voiture d'occasion!

C'est très révélateur ce que vous racontez. En fait, on s'aperçoit de deux choses : d'abord, que la cicatrice de la blessure encourue dans les camps n'est jamais sûre peut 
s'ouvrir à tout moment et avoir des conséquences irréversibles, même longtemps après la déportation. Comme lécrit Boris Cyrulnik, le traumatisme a constitué à un momen donné une brèche dans le développement de la personnalité et reste un point faible qui peut toujours se déchirer sous les coups du sort (Cyrulnik, 2004, p. 14), dans ce cas un échec professionnel cuisant associé à une perte financière importante et une perte de confiance de l'être aimé. Cette fêlure contraint le résilient à « travailler sans cesse à sa métamorphose interminable », à « tricoter sa résilience pendant tout le reste de sa vie ", à devenir a a te pas seurs à dever r ailleurs, on constate également quà un moment critique de votre existence, deux tuteurs de résilience externes et internes ont parfaitement fonctionné : d'une part, l'attachemen pour votre femme Nelly, d une part leffer de lextraordinaire force morale acquise duran la déportation et qui s'est avérée suffisante et nécessaire pour affronter cette nouvelle épreuve, rebondir et poursuivre votre processus de résilience.

J'aimerais parler aussi de Philippe Croizon que j’ai rencontré en 2012 sur un navire de plaisance lors d'une séance de plongée en Egypte. Comme vous le savez Philippe Croizon est un sportif de l'extrême amputé des deux bras et des deux jambes. À lâge de 26 ans, il a reçu une décharge de 20000 volts pendant qu'il enlevait une antenne de télévision sur le toit de sa maison. Il a eu les deuxbras etles deuxjambes anteńs de tén brûlés, qu'il a fallu amputer. Sa première reaction fut de ne pas vouloir vivre comme un légume. Avec un moral d'acier et une volonté de fer, il s'est battu pour vivre normalement, comme un être humain, pour être un exemple pour les handicapés. Il a réussi son pari. La preuve en est qu'il a fait la traversée du Détroit de Gibraltar et du Détroit de Behring en Alaska à la nage. Un film, Nager au-delà des frontières, a été tourné sur lui et diffusé dans l'émission Thalassa à l'occasion de la journée internationale des personnes handicapées. Le 10 janvier 2013, il a fait une plongée dans la fosse la plus profonde du monde à NEMO 33, c'est-à-dire à moins 33 mètres sous l'eau. Philippe donne de nombreuses conférences dans des sociétés, des usines, afin de montrer qu’un handicapé peutêtre productif pour autant qu'il soitconsidéré comme un être humain.

\section{Dans quelle mesure cette rencontre a-t-elle été importante pour vous?}

Après le décès de Nelly en 2012, j’avais perdu le goût de vivre. J’allais sur mes 86 ans et je n’imaginais pas la fin de ma vie sans elle à mes côtés. Je n’avais qu’une seule envie, lentement me laisser glisser... la rejoindre et dormir avec elle pour léternité. Cette rencontre fabuleuse aété pour moi un miracle, un signe du destin. Elle a remis en marche mon moteur créatif. J'ai entrepris de raconter plus en détail ma vie après Auschwitz et bien d'autres projets ont jailli alors dans mon esprit.

À mon sens, cette rencontre, qui a redonné du sens à votre vie, confirme bien le fait que le processus de résilience doit être régulièrement activé et entretenu par des tuteurs de résilience, que c'est un lent travail de cicatrisation, qui n'est jamais achevé.
Je pense en effet que si je n’avais pas rencontré Nelly, Jacques Meert et Philippe Croizon à des moments précis, ma vie se serait déroulée d'une tout autre manière et je crois que je n'aurais pas pu entamer ni achever ce que les psychologues appellent «mon processus de résilience».

Il est indéniable que vous avez fait preuve tout le long de votre carrière d'un grand dynamisme et d'une grande créativité. Cette créativité est interprétée par Boris $C$ yrulnik comme étant une arme dans le combat de la résilience contre le traum Selon lui, le comme ên tution du temps et lui a donné un « semblant d'urgence créatrice » (Cyrulnik, 2004, p. 141). Marie-Frédérique Bacque abonde dans ce sens; pour elle, labondante énergie vitale dont dispose le résilient est transformée, sublimée, et canalisée vers des activités de création (Bacqué). Pour certains, c'est le thêâtre, l'humour, l'intellectualisation, l'activité artistique, « tout ce qui permet d'intégrer le traumatisme, de digérer le malheur en le rendant familier et même agréable, une fois métamorphosé. „ (Cyrulnik, 2004, p. 140) Dans votre cas, cela a été la publicité, la communication, lévénementiel, la prospection touristique et hôtelière, les activités sportives, le témoignage et l'écriture, dont on reparlera plus tard. Chez vous, cette créativité se manifeste encore aujourd'hui ou je me trompe ?

En effet,j’ai encore une activité débordante. Mes journées passent très vite, car je suis toujours actif J'entretiens mon corps et mon esprit au mieux Lorsqu'une année suis toujours actif. J'entretiens mon corps et mon esprit au mieux. Lorsqu'une année scolaire redémarre, je reprends mes témoignages, environ quarante à cinquante par an, en Belgique et au Grand-Duché de Luxembourg, et j'effectue quelques voyages à Auschwitz. En tant que membre du Conseil d'Administration de la Fondation Auschwitzà Bruxelles et membre actif de la Commission pédagogique,j’ai participé durant un an aux travaux préparatoires à la mise sur pied du Train des 1000, ce train a emmené 1000 élèves pour effectuer un voyage en train de Bruxelles à AuschwitzBirkenau, afin qu'ils visitent le mémorial en compagnie de personnalités politiques. J'ai encore de nombreux projets, mon esprit bouillonne et je suis loin d’avoir mis la clé sous la porte. Que me réserventes quelques années qui me restent encore à la clé sor la porte Que ma vivre ? Je compte bien continuer à les employer en utilisant ma créativité et mon
imagination.

Pendant des années, vous n'avez pas parlé de votre expérience des camps, ni à vos enfants, ni à personne, comme nous l'avons déjà fait remarquer tout à l'heure. D'où ma question : qu'est-ce qui vous a décidé à rompre le silence, à témoigner et devenir passeur de mémoire?

En 1987, à Pâques, je me trouve à Auschwitz avec ma sœur Betsy. Nous faisons partie d'un voyage organisé par la Fondation Auschwitz de Bruxelles. C'est la première fois que je reviens dans ce lieu qui a vu périr mes parents et mon jeune frère mavid. Aussi, la visite est pour mi un calvaire Nous pomes une douza frère David. Aussi, la visite est pour moin calvaire. Nous some 
sur nos impressions, notre vie, notre parcours... Je suis mal à l'aise devant tous ces regards. Je me sens comme une bête curieuse, enfermée dans la cage d'un zoo avec d'autres bêtes rares. Je laisse les autres rescapés répondre aux questions. Certains n’hésitent pas à exagérer, faisant des amalgames entre ce qu'ils ont lu et leurs propres vécus. Le dernier jour, un des enseignants s'adresse directement à moi et me demande pourquoi je garde le silence. Alors, je prends la parole. C'était la première fois que je parlais en public. J'improvise et explique pourquoi la visite été très dure et pourqui on m'a demandé de témoigner. J'insiste sur le fait qu'on été très dure êt a assassine des millions de gens à Auschwitz et que les survivants doivent transmettre ce qu'ils ont vu et entendu. Tous les participants restent silencieux un lon moment. Puis, ils m’applaudissement longuement. J'étais très ému. Depuis, j’a appris à parler devant un public, essentiellement composé de jeunes. J’ai compris que c'est important que je témoigne. Comme l'écrit Elie Wiesel dans un article du Vif/L'Express du 6 janvier 2012 : «Porter témoignage, on ne le fait jamais assez. Je ne sais pas pourquoi j’ai survécu. Pour quelle raison l'ai-je mérité et pas un autre? Puisque c'est le cas, je dois en faire quelque chose. Tous les survivants de cette époque sont conscients de ce devoir de transmission. Il fut un temps où personne ne voulait nous écouter Aujourd’hui, il n’y a plus une école qui n’inclut pas la Shoah dans son programme d’études.»

\section{Quel message désirez-vous faire passer lors de ces témoignages?}

Je tente par mon témoignage de faire comprendre que la réussite n’est pas nécessairement liée à la possession des gadgets modernes, mais à l'exploitation de ses propres valeurs. Comme je l'écris dans mon ouvrage :

Je suis convaincu que tous, nous avons en nous-mêmes des potentiels à exploiter pour réussir dans la vie. Qưautour de nous, la chance existe: il faut avoir le courage de lattrape et de l'exploiter. Avec du travail et de la persévérance, un jeune peut aller bien plus bin quil ne le coit Simplem lui-même. Com lui-mêne. Conse comment il peut exploiter ses propes fors sop pour créer sa personnalité dans ce monde en perpétuelle mutation (Sobol, 2010, p. 152)

On perçoit bien là le discours d'un résilient, qui a exploité les valeurs qui étaient en lui pour survivre et a réussi, par la suite, à surmonter ses difficultés. Le témoignage lui-même fait partie aussi du processus de résilience. Permettez-moi de citer à nouveau Boris Cyrulnik : « Le blessé qui se fait une représentation de soi en relation avec les autres et se propose de la raconter échappe à la prison du passé. »( Cyrulnik, 2008, p. 191) Ces témoignages font clairement partie integranted émoignages font clairement parte incérante dune thérapie destinée à cicatriser les anciennes blessures, une maniere de plus être un épouvantail isolé - pour reprendre les humains. Vous avez, je pense, également témoigné dans un film.

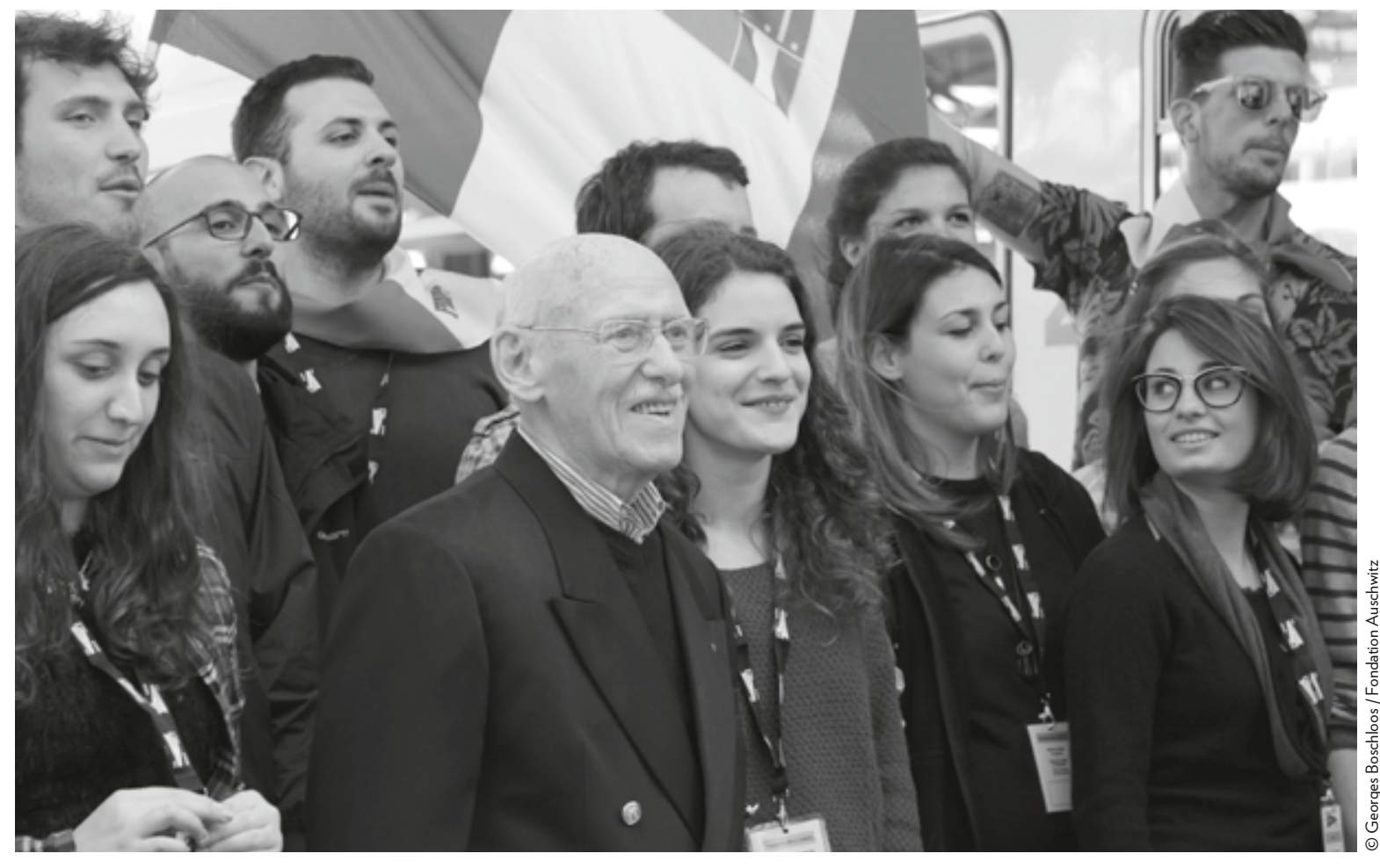

Oui, avec Sophie Rechtman dans le film Paul \& Sophie. Témoigner entre ombre et lumière, réalisé par Valentine Roels, d’après une idée d'Ina Van Looy du Centre Communautaire Laïc Juif (CCLJ). Il s'agit d'un film à vocation pédagogique qui donne la parole à Sophie Rechtman et à moi-même. Sophie a été cachée, enfant pendant plus de depx ans et a pu échap à à la déportation, grâce au coutenfant, , «Justes parmi les nations». Et moi, je suis le gamin de Bruxelles, le ketje, déporté à 18 ans, l'un des rares survivants. Le découpage en deux volets de témoignages eux-mêmes découpés en trois parties, avant, pendant et après la Shoah, facilite son utilisation par les enseignants et les animateurs. Par ailleurs, l'originalité du film est de dépasser le cadre classique des témoignages basé uniquement sur la description des faits. Cette mise en forme permet de mieux comprendre les émotions vécues par les témoins, voire de ressentir leur traumatisme.

Dans vos témoignages et dans ce film, vous faites ce qu'on peut appeler un véritable travail de mémoire.

Absolument. J’utilise le passé, en l'occurrence mon expérience dans les camps, pour une réflexion sur le présent et une projection vers l'avenir. «La mémoire c'est 
l'utilisation du passé pour une réflexion sur le présent et une projection vers l'avenir. La mémoire n’a de valeur que si elle se transforme en projet» a dit Paul Ricœur, donc il faut qu'elle nous projette dans le futur, qu'elle serve à l'amélioration de la vie humaine.

\section{Comment réagissent les jeunes, en général, devant ces témoignages?}

Très bien. Je leur demande de fermer les yeux pendant dix minutes en leur expliquant que, comme cela, le vieux bonhomme qui est devant eux disparaît et ils ne le voient plus! Mais ils entendent sa voix, et ils peuvent alors s'imaginer entendre un voient plus! Mais ils entendent sa voix, et ils peuvent alors s'imaginer entendre un
jeune de leur âge qui leur parle. Et quand je leur demande d'ouvrir les yeux, nous jeune de leur âge qui leur parle. Et quand je leur demande d'ouvrir les yeux, nous
sommes tous ensemble dans le camp d'extermination d'Auschwitz et ils ont tous fait, grâce à leur imagination, ce voyage. Je travaille avec les enseignants qui m'envoient les impressions de leurs élèves à propos de mes témoignages ; plus de $60 \%$ disent ainsi qu'ils ont apprécié cette technique de communication, qu'ils ont mieux compris la suite de mon parcours dans les camps de la mort.

J'imagine qu'ils vous posent aussi des questions. Que vous demandent-ils le plus souvent?

Ils me demandent pourquoi les nazis s’acharnaient sur les Juifs, sij’ai pardonné, ce que je dirai aujourd'hui, plus de soixante après, à un SS. Ils veulent savoir s'il y avait une certaine solidarité entre prisonniers, ce que sont devenus les Kapos, si j’ai gardé des contacts avec d'autres prisonniers, pourquoi je n’ai pas effacé mon tatouage, si aujourd'hui encore j’ai des cauchemars... Ils me posent des questions sur les conditions de vie dans les camps, le rôle de la Croix-Rouge, les « Marches de la Mort», les souffrances endurées.

Parlons à présent de l'écriture, qui est, selon Boris Cyrulnik, à côté du thêâtre, du dessin, du mot parlé une des manières de «maîtriser le sentiment d’être agressé, de dessin, du mot part renouer avec ses proches et de sur Or, vous avez mis par écrit vos souvenirs des camps et avez raconté ce qui sest passé après votre libération dans un livre intitulé « Je me souviens d'Auschwitz... De l'étoile de shérif à la croix de vie. „ Manifestement, l'écriture a constitué également pour vous un des clés de votre processus de résilience.

J'ai écrit ce livre avec beaucoup d'attention, en m’impliquant énormément.

C'est votre « exutoire », selon Philippe Croizon. En rapport avec la résilience, les psychologues écrivent que c'est (aussi) en partageant ses émotions et en élaborant son


vécu que le ésilient repren intelletue ildep capacité à rebondir (Hannah E., 5 mars 2013). Son histoire traumatisante, représentée et gauchie, devient socialement acceptable et même utile aux autres (Cyrulnik, 2008, p. 163). Les écrivains, du reste, aussi arrivent au même constat. Dans l'Écriture ou la vie, Jorge Semprun écrit que, quand un fracas chasse un homme de la condition humaine, quand il est indécent de parler et impossible de se taire, lécriture accorde un détour supportable. Il ne s'agit, pas, selon lui, de faire revenir le passé qui réveillerait la douleur, il s'agit de maîtriser le sentiment blessé et de le remanier pour en faire une action politique, philosophique ou artistique (Semprun, 1994, cité par Cyrulnik, 2008, p. 193). C'est, de toute éridence que vous faites en tém ignt pe lou génidence, ce quevous fàs

\section{BIBLIOGRAPHIE}

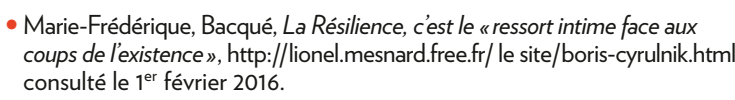
- Oscar, Chapital Colchado, «Résilience (psychologie),, in Encyclopédie 29 janvier 2016 .

- Philippe, Croizon (2014) : Plus fort la vie, Paris, Arthaud

- Boris, Cyrulnik (2004) : Les Vilians Petits Canards, Paris, Odile Jacob. - Boris, Cyrulnik (2008) : Autobiographie dun épouvantail, Paris, Odile - Boris, Cyrulnik (18 octobre 2010): : La honte caractérise la condition
humaine», Le Viffl'Express, p 8-12
-Boris, Cyrulnik (2012) : Sauve-toi, la vie táppelle, Paris, Odile Jacob.

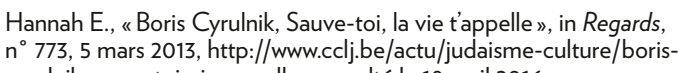

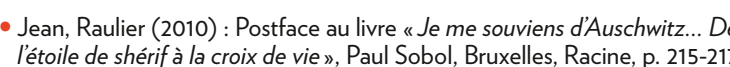
- Jorge, Semprun (1994) : LÉcriture ou la vie, Paris, Gallimard, «Folio ». - Paul, Sobol (2010) : Je me souviens d'Auschwitz... De létoile de shérifà Paul, Sobol (2010) : Je me souviens dAuschwitz... De letoile de sherrif à
la croid de vie, dossier pédagogique déric Lauwers, preface de Yannis
Thanassekos et Philippe Mesnard, postfface de Jean Raulier, Bruxelles, 